\title{
BMJ Open Enhancing peer support experience for patients discharged from acute psychiatric care: protocol for a randomised controlled pilot trial
}

\author{
Liana Urichuk, ${ }^{1,2}$ Marianne Hrabok, ${ }^{1,2}$ Katherine Hay, ${ }^{1}$ Pamela Spurvey, ${ }^{1}$ \\ Daniella Sosdjan, ${ }^{1}$ Michelle Knox, ${ }^{1}$ Allen Fu, ${ }^{2}$ Shireen Surood, ${ }^{1,3}$ Robert Brown, ${ }^{1}$ \\ Jeff Coulombe, ${ }^{1}$ Jill Kelland, ${ }^{1}$ Katherine Rittenbach, ${ }^{1,2}$ Mark Snaterse, ${ }^{1,2}$ \\ Adam Abba-Aji, ${ }^{1,2}$ Xin-Min Li, ${ }^{2}$ Pierre Chue, ${ }^{1,2}$ Andrew J Greenshaw, ${ }^{2}$ \\ Vincent I O Agyapong ${ }^{1,2}$
}

To cite: Urichuk L, Hrabok M, Hay $\mathrm{K}$, et al. Enhancing peer support experience for patients discharged from acute psychiatric care: protocol for a randomised controlled pilot trial. BMJ Open 2018:8:e022433. doi:10.1136/ bmjopen-2018-022433

- Prepublication history for this paper is available online. To view these files, please visit the journal online (http://dx.doi. org/10.1136/bmjopen-2018022433).

Received 17 February 2018 Revised 22 May 2018 Accepted 26 July 2018

Check for updates

(c) Author(s) (or their employer(s)) 2018. Re-use permitted under CC BY-NC. No commercial re-use. See rights and permissions. Published by BMJ.

${ }^{1}$ Addiction and Mental Health, Alberta Health Services, Edmonton, Alberta, Canada ${ }^{2}$ Department of Psychiatry, University of Alberta Faculty of Medicine and Dentistry, Edmonton, Alberta, Canada ${ }^{3}$ Faculty of Social Work, University of Calgary, Calgary, Alberta, Canada

Correspondence to Dr. Vincent I 0 Agyapong; agyapong@ualberta.ca

\section{ABSTRACT}

Introduction This study will evaluate the effectiveness of an innovative peer support programme. The programme incorporates leadership training, mentorship, recognition and reward systems for peer support workers, and supportive/reminder text messaging for patients discharged from acute (hospital) care. We hypothesise that patients enrolled in the peer support system plus daily supportive/reminder text messages condition will achieve superior outcomes in comparison to other groups.

Methods and analysis This is a prospective, raterblinded, four-arm randomised controlled trial. 180 patients discharged from acute psychiatric care in Edmonton, Alberta, Canada will be randomised to one of four conditions: (1) enrolment in a peer support system; (2) enrolment in a peer support system plus automated daily supportive/reminder text messages; (3) enrolment in automated daily supportive/reminder text messages alone; or (4) treatment as usual follow-up care. Patients in each group will complete evaluation measures (eg, recovery, general symptomatology and functional outcomes) at baseline, 6 months and 12 months. Patient service utilisation data and clinician-rated measures will also be used to gauge patient progress. Patient data will be analysed with descriptive statistics, repeated measures and correlational analyses. The peer support worker experience will be captured using qualitative methods.

Ethics and dissemination The study will be conducted in accordance with the Declaration of Helsinki (Hong Kong Amendment) and Good Clinical Practice (Canadian Guidelines). The study has received ethical clearance from the Health Ethics Research Board of the University of Alberta (Ref \# Pro00078427) and operational approval from our regional health authority (AHS- (PRJ) \#35293). All participants will provide informed consent prior to study inclusion. The results will be disseminated at several levels, including patients/peer supports, practitioners, academics/researchers, and healthcare organisations.

Trial registration number NCT03404882; Pre-results.

\section{INTRODUCTION}

\section{Background and rationale}

Peer support is emotional, social and practical help provided by non-professionals to assist people with sustaining health behaviours. ${ }^{1}$ Effective peer support workers share a similar condition as patients, are currently managing their condition effectively and have received training to provide support. ${ }^{2}$ Peer support can be provided to people with either psychological or non-psychological health complaints by peer supporter workers with 'lived' experience, and the position of peer support worker may include activities such as advocacy, connecting patients with resources and experiential sharing, among others. ${ }^{3}$

Peer support is consistent with the recovery paradigm in mental health, ${ }^{4}$ and is differentiated from psychiatric models of traditional diagnosis and treatment. The purported mechanisms through which peer support may function $^{2}$ are through experiential knowledge sharing, modelling of adaptive coping strategies, social comparison and enhancing social support. Moreover, peer support may be particularly useful for clients who have difficulty engaging in conventional services. ${ }^{1}$ It may be the case that peer support systems can serve as an entry point into the healthcare system for 'hardly reached' individuals and, at the very least, serve as a means of providing supportive services for those who would otherwise not engage in treatment. In addition, providing peer support may offer benefits to the peer supporter worker, by enhancing feelings of competence and personal value. ${ }^{2}$

Peer support is valued in recovery-oriented models $^{4}$ of mental health and is becoming 
increasingly implemented organisationally (see Myrick and del Vecchio ${ }^{5}$ for a discussion). Many studies have reported positive effects of peer support, including lower inpatient service use, better relationships with providers and increased engagement (for review, see Chinman et $\left.a t^{\dagger}\right)$. However, a recent rigorous evaluation of randomised controlled trials (RCTs) ${ }^{7}$ of peer support studies reported that outcomes were generally mixed and often non-significant. In their review, the authors noted a high degree of bias and methodological limitations in the studies they reviewed, and concluded that 'peer support programs should be implemented within the context of high quality research projects wherever possible'.

In addition to methodological improvements of research on peer support, there is a need to further develop the peer support workforce. Identified areas of development for peer support programmes include ${ }^{8}$ : how to define 'peerness', role clarity and integration with existing systems, credentialing,and workforce development (see also Myrick and del Vecchio ${ }^{5}$ ). Within our local context, in the province of Alberta, Canada, peer support for patients has been in existence for many years. Anecdotal evidence suggests there is low uptake (ie, number of programmes employing peer support workers and difficulty recruiting to available positions) and high attrition for peer support worker roles within the province. There has been a drive to develop curricula and organisational infrastructure to formally train and support peer support workers. Administrators who have worked with peer support workers suggest the lack of adequate incentives may contribute to peer support workforce challenges. Similarly, there are often limited incentives for mental health professionals to provide supervision and mentorship for peer support workers locally.

In summary, the existing literature suggests there is opportunity for innovation in peer support programmes, and there is a need for rigorous methodology to better evaluate outcomes from peer support programmes. There is also a need to further develop the peer support workforce. The current paper describes an RCT that is designed to systematically evaluate a novel peer support model termed the 'Edmonton Peer Support System' (EPSS), which incorporates innovative features of service delivery (ie, an incentive-based model of training, communication with patients via text) and rigorous methodological design to minimise bias and confounds (eg, comprehensive measures of outcome; methodological design features including longitudinal, prospective, four-arm parallel design and rater blinding). Because we will be using paid peer support workers for this project, the role of incentives will focus on workforce development and reduction of attrition (vs enhancing recruitment).

One of the innovative features of this study is the incorporation of automated daily supportive text messaging. Text messaging is a relatively low-cost, high-impact and easily scalable programme that uses existing technology, is devoid of geographic barriers and is easily accessible to end users. There is evidence that supportive text messaging demonstrates positive effects in terms of symptom improvement and patient satisfaction. For example, patients with depression and comorbid alcohol use disorder showed significantly lower depressive symptoms on standardised self-report than a similar patient group who did not receive messages (large effect size). ${ }^{9}$ A second randomised trial demonstrated similar results (Cohen's $d=0.67$ ).$^{10}$ In terms of satisfaction, over $80 \%$ of subscribers reported that a texting programme improved their mental health. ${ }^{1112}$

There is also evidence that reminder text messaging improves appointment attendance rates. For example, in a systematic review and meta-analysis of eight RCTs involving 6615 participants, ${ }^{13}$ the authors found moderate quality evidence from seven studies (5841 participants) that mobile text message reminders improved the rate of attendance at healthcare appointments compared with no reminders (risk ratio (RR) 1.14; $95 \%$ CI 1.03 to 1.26 ). They also found that text messaging reminders were similar to telephone reminders in terms of their effect on attendance rates, yet cost less than telephone reminders.

Another innovative feature of this study is the incorporation of a peer support incentive system. As discussed, the peer support workforce has been identified as an area in need of development, ${ }^{5}$ including role clarity, integration with healthcare professionals and credentialing. ${ }^{8}$ Specifically, a lack of a 'career ladder' or a clear movement within positions of peer support work has been identified, ${ }^{8}$ with movement possible laterally (but not upward) within many programme structures. In this project, peer support workers will participate in an incentive-based system that formally recognises performance. The EPSS is structured as follows:

- Peer support workers are people with 'lived' mental health and substance abuse experience who are at a point in their personal recovery where they consistently demonstrate the ability to share their individual story in a manner that inspires hope and builds relationships with others. Peer support workers who are employed by Alberta Health Services (AHS), our provincial health authority, will be invited to enrol as foundational members of the EPSS.

- Members will be provided with formalised training developed within our health region, involving knowledge training, practice and ongoing meetings throughout offering the programme to assist peer support workers in adhering to principles consistent with a peer support framework and recovery philosophy and problem-solving concerns or challenges that may arise. Peer support workers will receive clinical support/mentorship from mental health therapists.

- Patient beneficiaries of EPSS will receive supportive face-to-face visits and interactive phone calls/texts from their peer support workers.

- Interested patient beneficiaries will be enrolled as associate members of the EPSS and will be provided with training to act as peer support workers when they are at an advanced stage in their recovery. At this time, 
they can apply for AHS peer support worker roles as available.

- Contributions of peer support workers will be recognised via attainment of different levels of membership (eg, 'star' levels) and certificates of recognition (bearing logos of collaborating institutions) at formal ceremonies. Star-level recognition will be categorised into: Silver Star Memberships (5 stars), Gold Star Memberships (10 stars) and Star Fellowships (20 stars).

- Contributions of mental health therapists towards supporting/mentoring peer support workers will also be recognised through star memberships and certificates at formal ceremonies.

The hope of EPSS is to increase compassion and decrease stigma, foster multidisciplinary teamwork, incorporate client/caregiver experiences and strengthen service provider skills and abilities. Our proposed EPSS will provide training for those with mental health challenges that will enable them to contribute to the provision of compassionate care for mental health patients. This programme philosophy is aligned with regional goals relating to improvement of mental health services in our province. ${ }^{14}$

\section{Aim and objectives}

The aim of the pilot project is to evaluate the feasibility of implementing the EPSS and/or the automated daily supportive text message service for patients discharged from acute care. Because of the limited availability of data in this specific area, another aim is to generate effect size data for these interventions which will help in sample size and power calculations for a full trial. The client outcomes are organised according to: recovery variables (eg, recovery, stigma), functional variables (quality of life, employment), symptom variables (psychological symptoms, general outcomes) and secondary outcomes will be service variables (eg, heath service utilisation, cost, satisfaction), as further described in Methods and analysis section. Peer support worker outcomes will also be evaluated, including effectiveness, job satisfaction, acceptability and recovery (both patient and peer support worker self-recovery). For clients, the two symptom variables (Health of Nations Outcome Scale (HoNOS) and Behaviour and Symptom Identification Scale (BASIS-32) will be the primary outcomes and all other measures will be secondary outcomes.

The objectives of the project include:

1. To assess ease of recruitment and follow-up of patients in the study and to test the success of blinding of outcome assessors.

2. To compare mean changes in recovery, functional outcomes, clinical symptoms and service variables after 6 and 12 months for patients in each of the four arms: those discharged from acute care and enrolled in peer support system, those enrolled in peer support system plus daily supportive/reminder text messages, those receiving supportive/reminder text mes- sages but no peer support and those receiving usual follow-up care.

3. To assess job satisfaction, acceptability and recovery of peer support workers at 6 and 12 months.

4. To assess job satisfaction among mental health therapists offering support and mentorship for peer support workers.

\section{Hypothesis}

We hypothesise that for the full study, patients enrolled in the peer support system plus automated daily supportive/ reminder text messages will achieve superior outcomes compared with other conditions on each outcome measure used. In turn, patients enrolled in the peer support-only arm and those enrolled in the automated daily supportive text message-only arm will have superior outcomes to those enrolled in the treatment as-usual arm.

\section{METHODS AND ANALYSIS}

\section{Overview of study design, timeline and participant selection}

This will be a longitudinal, prospective, parallel design, four-arm, rater-blinded RCT with a recruitment period of 6 months and an observation period of 12 months for each participant. Table 1 provides an overview of the timeline for the project. The research will be carried out in Addiction and Mental Health clinics in a large, sociodemographically diverse ${ }^{15}$ city in Western Canada (Edmonton, Alberta).

Patients who are 18 to 65 years of age, able to provide informed written consent, have been diagnosed with an enduring mental health condition and are ready for discharge from an acute care facility within the Edmonton Zone with community mental health follow-up will be invited to participate. Patients should have a mobile handset capable of receiving text messages. Mobile phones and a call credit of up to 10 dollars monthly will be provided to those who satisfy the inclusion criteria but do not have mobile phones. Patients will be ineligible if they do not meet the above inclusion criteria, if they have an addiction disorder but not a mental health diagnosis, are not capable of reading text messages from a mobile device or if they know they will be out of town during the 12-month follow-up period. Patients are also ineligible if they do not consent to take part in the study.

\section{Interventions}

Each of the four intervention arms will be randomly assigned. Guidance of the peer support structure will be derived from the Mental Health Commission of Canada Guideline for the practice and Training of Peer Support (2016). While each peer-patient meeting will be fluid and tailored individually for the priorities identified at each visit, the core values of sharing lived experience, promoting self-determination and establishing an empathetic relationship will be applied. The nature of automated daily text messages will be derived from a database of established phrases related to various psychiatric disease 
Table 1 Gantt chart timeline

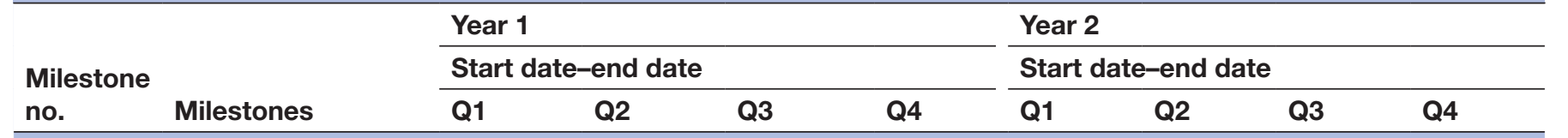

Milestone 1: Recruiting and training of peer support workers and trainee in psychiatry

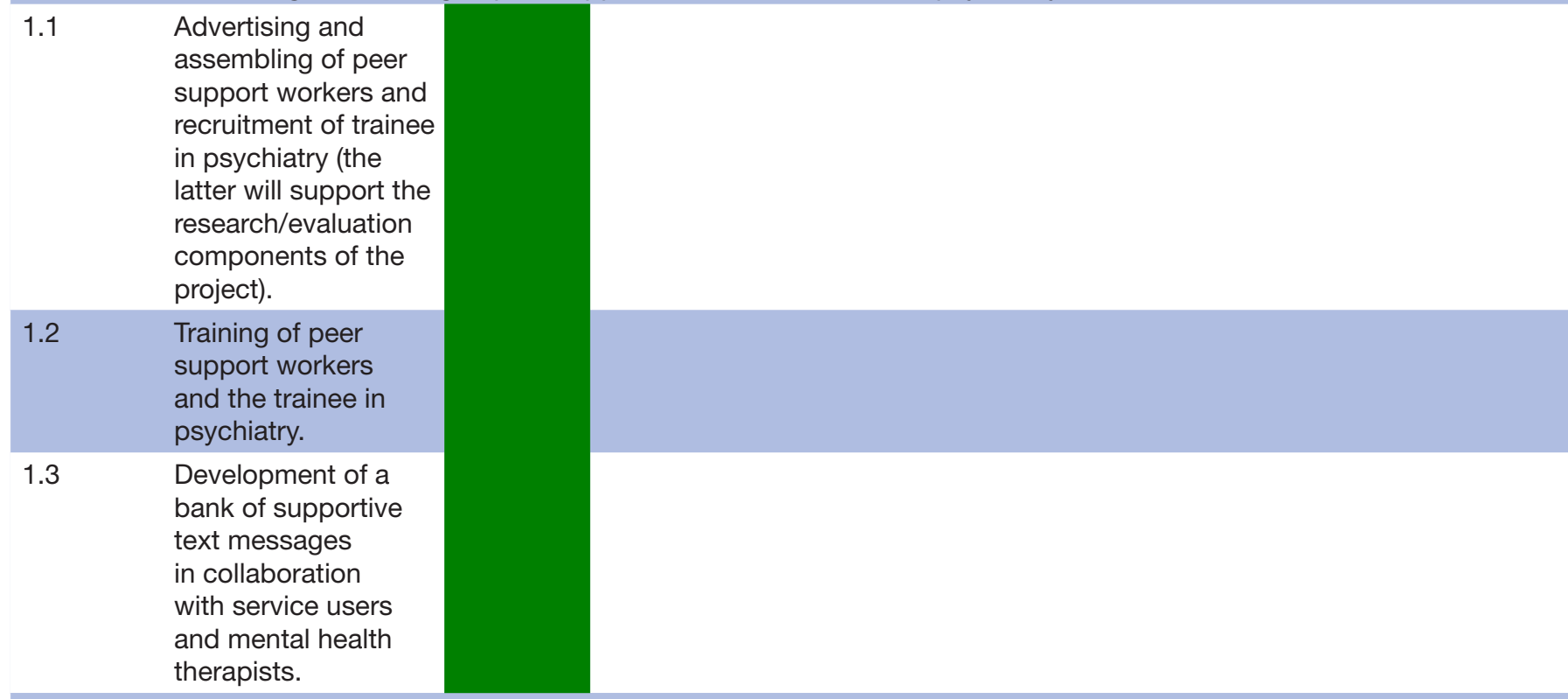

Milestone 2: The recruitment of study participants

$\begin{array}{ll} & \text { Recruitment, } \\ \text { baseline assessment, }\end{array}$ randomisation.

2.2 Assignment into one of the four arms of the study.

2.3 Provision of peer support and delivery of supportive text messages to participants in three arms of the study (ie, not the usual care group).

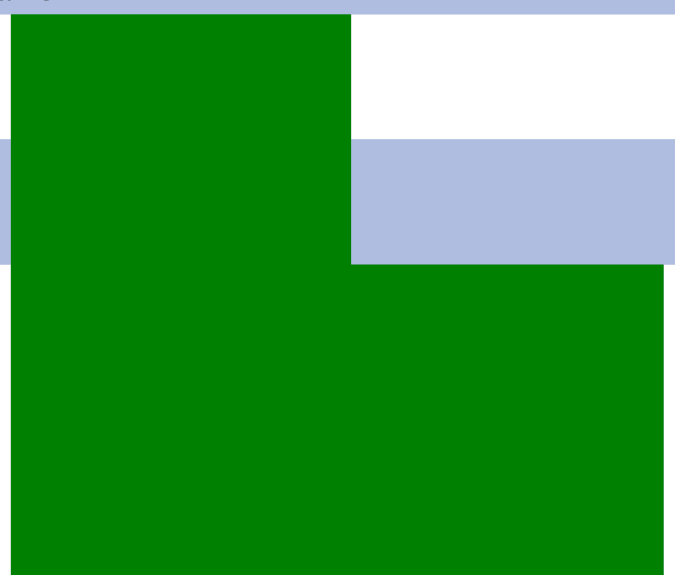

Milestone 3: Follow-up assessment of study participants and collection of administrative data

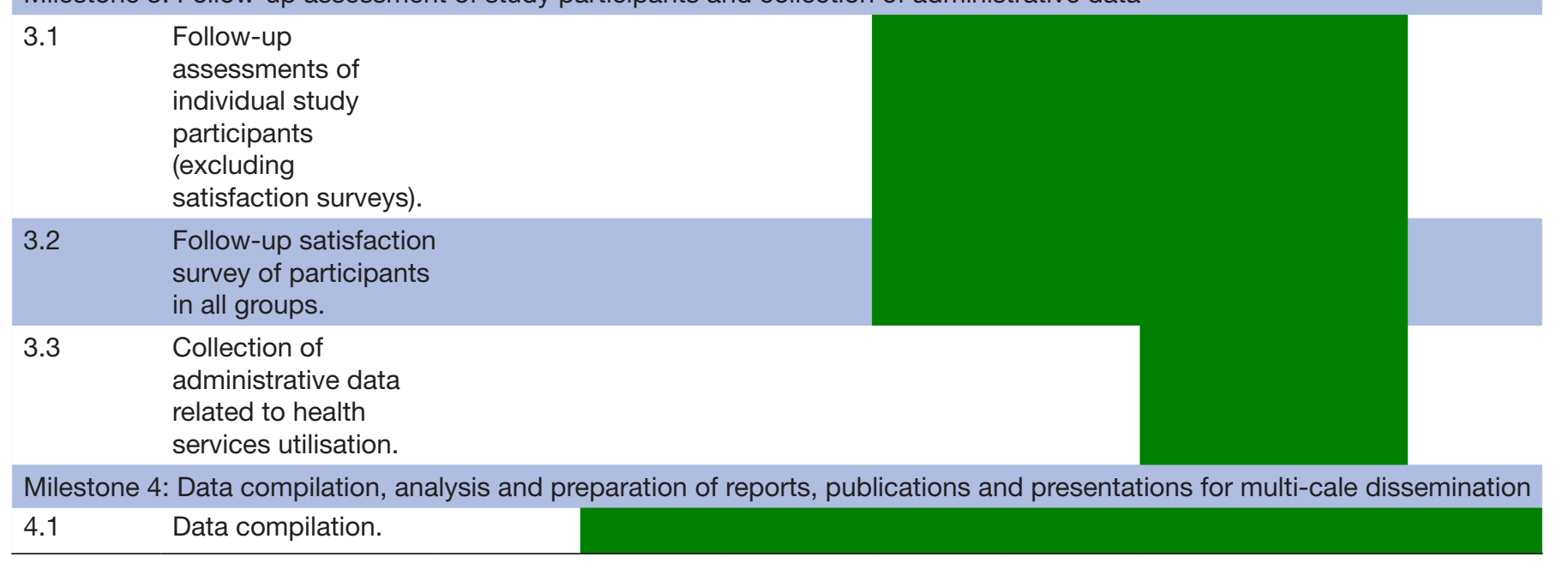




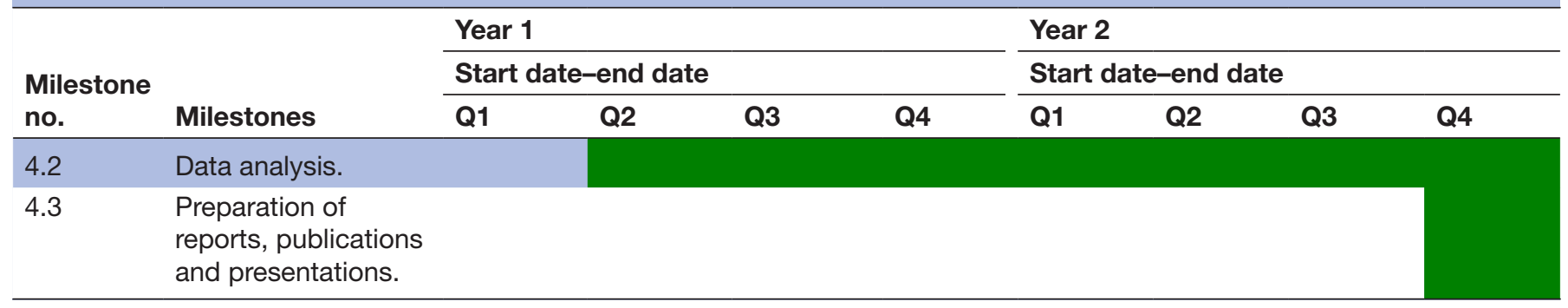

groupings for personalised and applicable support. Thus, automated daily text messages will be comprised of condition-specific symptom management content (eg, activity scheduling in depression, importance of medication adherence) and general, widely indicated and symptomatology-independent content (eg, self-care, social support, hope, affirmation and recovery content).

1. Patients in the peer support system-only arm of the study will be assigned a peer support worker who will visit them during the last week of their inpatient stay to introduce themselves and build rapport before patients are discharged into the community. The peer support workers will visit the participants up to eight times over a 6 -month period. The peer support workers will offer the opportunity for interactive phone calls/texts between themselves and the patients for 6 months, but patients will not receive automated daily supportive text messages from an online application nor will they receive reminder text messages for their community clinic/programme appointments. Patients will continue to receive whatever community clinic/ programme treatment options they are provided.

2. In the peer support system plus automated daily supportive/reminder text messages condition, patients will be assigned a peer support worker (as described above) and will receive up to eight visits over a 6-month period, in addition to whatever community clinic/ programme treatment options they are provided. In addition to peer support, participants in this arm of the study will receive automated daily supportive text messages from an online application and reminder text messages for their community clinic/programme appointments.

3. Patients in the automated daily supportive/reminder text messages alone-only arm of the study will receive automated daily supportive text messages from an online application and the message content will be aligned with their current areas of concern. Reminder text messages for their community clinic/programme appointments will also be provided.

4. Patients in the treatment-as usual follow-up care (ie, control arm) of the study will receive the usual follow-up appointment offered to all patients who are discharged from acute care. They may also be offered a range of community clinic/programme treatment options. However, they will receive neither peer support nor automated daily supportive/reminder text messages.

Only patients in the peer support arms of the study (ie, numbers 1 and 2, above) will be offered affiliate membership of the EPSS. However, patients in all arms of the study can enrol as members of the EPSS when they are at an advanced stage in their recovery and are successful in securing peer worker positions within the health service.

\section{Sample size}

Consistent with the idea that this is a pilot study, with no established effect size data available to aid in power and sample size calculations, the research will use data that can be elicited from participants who can be enrolled within existing operational resources. This method is acceptable for pilot studies involving novel interventions and have been described by Haynes et al as using 'the patients I can get'. ${ }^{16}$ The study will therefore be limited to a sample size of 180 , with about 45 patients recruited into each arm of the study. Patients transitioning from acute care are vulnerable to insufficient service follow-up postdischarge, and it can reasonably be expected that only a small number of eligible participants will enrol in and complete the study.

\section{Outcomes}

Outcome measures and time points are detailed in tables 2 and 3, and follow from the aim and objectives of the study. All measures (with the exception of patient experience questionnaire, interviews and data extraction) are objective measures with published information regarding reliability and validity.

The two symptom variables (HoNOS and Basis-32) will be the primary outcomes and all other measures will be secondary outcomes.

\section{Randomisation and blinding}

Randomisation will be stratified by primary mental health diagnoses (mood or psychotic disorder) using permuted blocks to ensure balance $(1: 1: 1: 1)$ between the four follow-up community treatment groups. The randomisation codes will be transmitted by an independent statistician via text message directly to a researcher's password-protected phone line with a secure online backup. This will commence as soon as participants sign the consent forms. 
Table 2 Client-oriented Outcome measures

\begin{tabular}{|c|c|c|c|c|c|c|c|}
\hline & \multirow[b]{2}{*}{ Construct } & \multirow[b]{2}{*}{ Tool } & \multirow[b]{2}{*}{ Rater } & \multirow{2}{*}{$\begin{array}{l}\text { Time } \\
\text { required }\end{array}$} & \multicolumn{3}{|c|}{ Timepoints assessed } \\
\hline & & & & & Baseline & 6 months & 12 months \\
\hline $\begin{array}{l}\text { Recovery } \\
\text { variables }\end{array}$ & Recovery & Recovery Assessment Scale ${ }^{19}$ & Client & $5 \min$ & $x$ & $x$ & $x$ \\
\hline \multirow[t]{2}{*}{$\begin{array}{l}\text { Functional } \\
\text { variable }\end{array}$} & Quality of life & $\begin{array}{l}\text { WHOQOL-BREF (World } \\
\text { Health Organisation Quality of } \\
\text { Life Brief instrument) }\end{array}$ & Client & $10 \mathrm{~min}$ & $x$ & $x$ & $x$ \\
\hline & Employment & Employed $^{*}$ yes/no & Client & $1 \mathrm{~min}$ & $x$ & $x$ & $x$ \\
\hline \multirow[t]{2}{*}{$\begin{array}{l}\text { Symptom } \\
\text { variables }\end{array}$} & $\begin{array}{l}\text { Overall } \\
\text { outcomes }\end{array}$ & $\begin{array}{l}\text { HoNOS (Health of Nations } \\
\text { Outcome Scale) }\end{array}$ & Clinician & $5 \min$ & $X$ & $x$ & $X$ \\
\hline & $\begin{array}{l}\text { Overall } \\
\text { symptoms }\end{array}$ & $\begin{array}{l}\text { BASIS-32 (Behaviour and } \\
\text { Symptom Identification } \\
\text { Scale) }\end{array}$ & Client & $10 \min$ & $x$ & $x$ & $x$ \\
\hline & $\begin{array}{l}\text { Health } \\
\text { services cost }\end{array}$ & Costs of above services & Researcher & - & & & $x$ \\
\hline & $\begin{array}{l}\text { Patient } \\
\text { satisfaction } \\
\text { with service }\end{array}$ & $\begin{array}{l}\text { Patient Satisfaction/ } \\
\text { Experience Questionnaire }\end{array}$ & Client & $5 \mathrm{~min}$ & & $x$ & $x$ \\
\hline & & & Total time & Client & $\sim 30 \mathrm{~min}$ & $\sim 35 \mathrm{~min}$ & $\sim 35 \mathrm{~min}$ \\
\hline & & & & Clinician & $\sim 5 \min$ & $\sim 5 \mathrm{~min}$ & $\sim 5 \min$ \\
\hline
\end{tabular}

*For example, paid full time, paid part time, self-employed, paid casual, temporary or contract work, unemployed, volunteering. †Fr example, rates of use, inpatient admissions and length of stay, readmissions, completed appointments, emergency department presentations, emergency medical services use, community services appointments, crisis and urgent service calls, no show rates.

Table 3 Peer support worker-oriented outcome measures

\begin{tabular}{|c|c|c|c|c|c|c|}
\hline \multirow[b]{2}{*}{ Construct } & \multirow[b]{2}{*}{ Instrument } & \multirow[b]{2}{*}{ Rater } & \multirow[b]{2}{*}{ Time required } & \multicolumn{3}{|c|}{ Timepoints assessed } \\
\hline & & & & Baseline & 6 months & 12 months \\
\hline Effectiveness & $\begin{array}{l}\text { Interview (part a)* } \\
\text { Data extraction and } \\
\text { analysis, including: number } \\
\text { of interactive phone calls/ } \\
\text { texts per patient, number } \\
\text { of visits provided per } \\
\text { patient† }\end{array}$ & $\begin{array}{l}\text { Researcher with } \\
\text { peer support } \\
\text { worker } \\
\text { Researcher }\end{array}$ & $10 \min$ & & $x$ & $\begin{array}{l}X \\
X\end{array}$ \\
\hline Job satisfaction $\ddagger$ & Interview (part b) & $\begin{array}{l}\text { Researcher with } \\
\text { peer support } \\
\text { worker }\end{array}$ & $5 \mathrm{~min}$ & & $x$ & $x$ \\
\hline Acceptability & Interview (part b) & $\begin{array}{l}\text { Researcher with } \\
\text { peer support } \\
\text { worker }\end{array}$ & $10 \min$ & & $x$ & $x$ \\
\hline Recovery & & Total time & $\begin{array}{l}\text { Peer support } \\
\text { worker }\end{array}$ & $5 \mathrm{~min}$ & $20 \min$ & $30 \mathrm{~min}$ \\
\hline
\end{tabular}

*Includes questions regarding: amount of on the job training; frequency and type of activities and support (received and given); perceptions of accountability, empowerment, self-confidence, self-awareness, self-esteem; satisfaction with support received; role clarity; personal wellbeing; levels of comfort and team integration.

†For example, number of interactive phone calls/texts sent to/received by each patient, number of peer support visits per patient, peer support worked attrition rates (pre and post)

¥Mental health therapists providing supervision for peer workers will also be interviewed regarding their job satisfaction 
Because it will not be possible for participants to be blinded, treatment allocation will be made explicit to them as soon as randomisation is concluded. Outcome assessors will be blinded to treatment group allocation by not involving them in discussions about study participants and not granting them access to the database which contains the randomisation code. In addition, study participants will be asked not to reveal their treatment allocation to their assessor. Moreover, these assessors will not be involved in data analysis. To test the success of blinding, we will ask the assessor to guess the treatment group for each participant at 6-month and 12-month follow-up. After data collection is complete, all data will undergo a blind review for the purposes of finalising the planned analysis.

\section{Follow-up assessment}

At 6 and 12 months, a blinded researcher will contact all study participants and assist them to complete a range of assessment tools relating to the primary and secondary outcome measures. They will be offered the opportunity to complete the assessments face to face or over the phone. At 12 months, data related to each person's clinic/ programme attendance rates and utilisation of health services will be compiled from administrative records by the blinded researcher. A researcher will also contact peer supporters and mental health therapists to determine their experience regarding the training process and meetings. Their perception of their job satisfaction (eg, self-confidence, self-esteem, etc) and job acceptability will be recorded with a constant comparative analysis approach to complete exploratory outcome measures. Attrition rates for peer support workers participating in the EPSS will also be recorded. At the end of the study, focus group workshop will also be organised for a cross section of patients in all four arms to obtain qualitative feedback about their follow-up experience.

\section{Statistical methods}

The primary goal of the statistical analysis will be to produce summary descriptive statistics for the longitudinal data, which will provide estimates for future sample size calculations and enable calculation of effect size. Another goal will be to estimate the variability between the results and what has been stated by our hypothesis for the full study to represent a clinically meaningful difference between the intervention and control groups. The data will be analysed using repeated measures and effect size analyses, and correlational analyses will be completed between measures at each time point. The results of this study will guide the design for a future, more highly powered, study. Quantitative data collected from peer support workers and mental health therapists will be analysed using descriptive analysis. Inferential statistics will be used to compare attrition rates for EPSS peers with those of peers who worked within the Edmonton zone AHS 2 years prior to the launch of the EPSS. Qualitative data collected from interviews with peer support workers and mental health therapists as well as the focus group workshop with patients will be analysed thematically using the NVivo software.

\section{Patient and public involvement}

This study was conceived and designed to address gaps in the care/support available at the community level for psychiatric patients who have been discharged from hospital and referred to community mental health services for follow-up. The study was designed with the active involvement of a patient representative who is a coauthor of this protocol. Peer support workers who are essentially patients would play central roles in this study, including delivering part of the interventions proposed in this protocol. Our randomised trial offers patients the opportunity to provide feedback regarding the burden of the intervention through a focused group workshop involving a cross section of patients in all arms of the study.

\section{ETHICS AND DISSEMINATION}

The study will be conducted in accordance with the Declaration of Helsinki (Hong Kong

Amendment) ${ }^{17}$ and Good Clinical Practice (Canadian Guidelines).$^{18}$ The study has received ethical clearance (Ref \# Pro00078427) and operational approval from our regional health authority (AHS-(PRJ) \#35293). The study is registered with clinicaltrials.gov: NCT03404882. All participants will provide informed consent prior to study inclusion.

The results of the study are expected to be available within 21 months of project commencement. We intend to disseminate the research findings at several levels, including patients/peer supports, practitioners, academics/researchers and healthcare organisations. Patients involved with the research will be invited to help develop key messages arising from the work and help to determine the format and modality for dissemination of findings. We will use existing communication channels within our health authority to disseminate findings to clinicians. We will disseminate findings to academics and researchers through peer-reviewed journals and provincial, national and international academic forums.

\section{DISCUSSION}

The results of the study will provide important information with respect to: a comprehensive evaluation of outcomes of peer support; comparability of peer support compared with care as usual; impact of automated daily supportive/reminder text messaging in addition to care; and impact of a novel, incentive-based peer support training system. The results of the pilot trial will also inform the implementation of a multicentre clinical trial which may be necessary to validate our study findings. Given the increasing implementation of peer support programmes within service organisations, the results of 
this study will be useful for administrators and clinicians who are interested in incorporating peer support services into existing care.

Although this is a pilot trial, the highest quality design was chosen to evaluate the EPSS. We therefore expect that the pilot findings will inform and support administrative decision-making with respect to further scaling and studying the intervention within the province of Alberta and beyond. Thus, we will plan an organisational engagement strategy to advance discussions about feasibility and effectiveness prior to the conclusion of the trial. This will help ensure the findings are a relevant part of decision-making processes in a way that is aligned with study findings as they emerge. This may facilitate planning of a larger study that is endorsed at both leadership and operational levels, so that the potential impact of the intervention can reach patients in a more timely fashion.

Contributors The study was conceived and designed by VIOA who also contributed to drafting of the initial and final drafts of the manuscript. LU, MH and $\mathrm{KH}$ contributed to the study design and drafting of the initial and final drafts of the manuscript. PS, DS, MK, AF, SS, RB, JC, JK, KR, MS, AA-A, X-ML, PC and AJG contributed to the study design and revising the initial draft of the of the manuscript. All authors approved of the final draft of the manuscript prior to submission.

Funding This work was supported by the Douglas Harding Trust (via Department of Psychiatry, University of Alberta) and support was received from Alberta Health Services.

Competing interests $\mathrm{MH}$ receives royaltiesfrom 0xford University Press. The other authors have nothing to disclose.

Patient consent Not required.

Ethics approval Health Ethics Research Board of the University of Alberta (Ref \# Pro00078427)

Provenance and peer review Not commissioned; externally peer reviewed.

Data sharing statement N/A as this is only a protocol.

Open access This is an open access article distributed in accordance with the Creative Commons Attribution Non Commercial (CC BY-NC 4.0) license, which permits others to distribute, remix, adapt, build upon this work non-commercially, and license their derivative works on different terms, provided the original work is properly cited, appropriate credit is given, any changes made indicated, and the use is non-commercial. See: http://creativecommons.org/licenses/by-nc/4.0/.

\section{REFERENCES}

1. Sokol R, Fisher E. Peer support for the hardly reached: a systematic review. Am J Public Health 2016;106:1308 https://ajph. aphapublications.org/doi/full/.

2. Proudfoot JG, Jayawant A, Whitton AE, et al. Mechanisms underpinning effective peer support: a qualitative analysis of interactions between expert peers and patients newly-diagnosed with bipolar disorder. BMC Psychiatry 2012;12:196.

3. Jacobson N, Trojanowski L, Dewa CS. What do peer support workers do? A job description. BMC Health Serv Res 2012;12:205.

4. Onken SJ, Craig CM, Ridgway P, et al. An analysis of the definitions and elements of recovery: a review of the literature. Psychiatr Rehabil J 2007:31:9-22.

5. Myrick K, Del Vecchio P. Peer support services in the behavioral healthcare workforce: State of the field. Psychiatr Rehabil J 2016;39:197-203.

6. Chinman M, George P, Dougherty RH, et al. Peer support services for individuals with serious mental illnesses: assessing the evidence. Psychiatr Serv 2014;65:429-41.

7. Lloyd-Evans B, Mayo-Wilson E, Harrison B, et al. A systematic review and meta-analysis of randomised controlled trials of peer support for people with severe mental illness. BMC Psychiatry2014;14:39 http:// bmcpsychiatry.biomedcentral.com/articles/.

8. Silver J, Nemec PB. The role of the peer specialists: Unanswered questions. Psychiatr Rehabil J 2016;39:289-91.

9. Agyapong VI, Ahern S, McLoughlin DM, et al. Supportive text messaging for depression and comorbid alcohol use disorder: singleblind randomised trial. J Affect Disord 2012;141(2-3):168-76.

10. Agyapong $\mathrm{VIO}$, Juhás $\mathrm{M}$, Ohinmaa $\mathrm{A}$, et al. Randomized controlled pilot trial of supportive text messages for patients with depression. BMC Psychiatry 2017;17:286.

11. Agyapong VI, McLoughlin DM, Farren CK. Six-months outcomes of a randomised trial of supportive text messaging for depression and comorbid alcohol use disorder. J Affect Disord 2013;151:100-4.

12. Agyapong VI, Mrklas $\mathrm{K}$, Juhás $\mathrm{M}$, et al. Cross-sectional survey evaluating Text4Mood: mobile health program to reduce psychological treatment gap in mental healthcare in Alberta through daily supportive text messages. BMC Psychiatry2016;16:378 http:// bmcpsychiatry.biomedcentral.com/articles/.

13. Gurol-Urganci I, de Jongh T, Vodopivec-Jamsek V, et al. Mobile phone messaging reminders for attendance at healthcare appointments. Cochrane Database Syst Rev 2013;12:CD007458.

14. AAH-G. Valuing mental health: Next steps: Alberta Health website. http://www.health.alberta.ca/initiatives/Mental-Health-Review.html (cited 3 Jan 2018).

15. Edmonton c of. 2016 municipal census results. $2017 \mathrm{https} / / / \mathrm{www}$. edmonton.ca/city_government/facts_figures/municipal-censusresults.aspx (cited 14 Sep 2017).

16. Haynes RB, Guyatt G, Tugwell P. Clinical Epidemiology: How to do Clinical Practice Research. Philadelphia PA: Lippincott, Williams, Wilkins, 2005.

17. World Medical Association. World Medical Association Declaration of Helsinki: ethical principles for medical research involving human subjects. JAMA 2013;310:2191-4.

18. WHO Technical Report Series. Guidelines for Good Clinical Practice (GCP) for Trials on Pharmaceutical Products, 1993. http://apps.who. int/medicinedocs/en/d/Jwhozip13e/. (cited 22 Nov 2017).

19. Giffort D, Schmook A, Woody C, et al. Recovery Assessment Scale. Cambridge MA: Human Services Research Institute, 1995.

20. Major B, O'Brien LT. The social psychology of stigma. Annu Rev Psychol 2005;56:393-421.

21. Skevington SM, Lotfy M, O'Connell KA; WHOQOL Group. The World Health Organization's WHOQOL-BREF quality of life assessment: psychometric properties and results of the international field trial. A report from the WHOQOL group. Qual Life Res 2004;13:299-310.

22. What is HoNOS?. http://www.rcpsych.ac.uk/traininpsychiatry/ conferencestraining/resources/honos/whatishonos.aspx (cited 2018 Jan 4).

23. Eisen SV, Normand SL, Belanger AJ, et al. The Revised Behavior and Symptom Identification Scale (BASIS-R): reliability and validity. Med Care 2004;42:1230-41. 